\title{
FIXED POINT THEOREMS FOR GENERALIZED LIPSCHITZIAN SEMIGROUPS IN BANACH SPACES
}

\author{
BALWANT SINGH THAKUR and JONG SOO JUNG
}

(Received 12 February 1997 and in revised form 22 July 1997)

\begin{abstract}
Fixed point theorems for generalized Lipschitzian semigroups are proved in $p$ uniformly convex Banach spaces and in uniformly convex Banach spaces. As applications, its corollaries are given in a Hilbert space, in $L^{p}$ spaces, in Hardy space $H^{p}$, and in Sobolev spaces $H^{k, p}$, for $1<p<\infty$ and $k \geq 0$.
\end{abstract}

Keywords and phrases. Semitopological semigroup, submean, generalized Lipschitzian semigroup, $p$-uniformly convex Banach space, uniformly normal structure.

1991 Mathematics Subject Classification. 47H10.

1. Introduction. Let $K$ be a nonempty closed convex subset of a Banach space $E$. A mapping $T: K \longrightarrow K$ is said to be Lipschitzian mapping if for each $n \geq 1$, there exists a positive real number $k_{n}$ such that

$$
\left\|T^{n} x-T^{n} y\right\| \leq k_{n}\|x-y\|
$$

for all $x, y$ in $K$. A Lipschitzian mapping is said to be nonexpansive if $k_{n}=1$ for all $n \geq$ 1 , uniformly $k$-Lipschitzian if $k_{n}=k$ for all $n \geq 1$, and asymptotically nonexpansive if $\lim _{n} k_{n}=1$, respectively. These mappings were first studied by Geobel and Kirk [6] and Geobel, Kirk, and Thele [8]. Lifshitz [10] showed that in a Hilbert space $H$, a uniformly $k$-Lipschitzian mapping $T$ with $k<\sqrt{2}$ has a fixed point. Downing and Ray [3] and Ishihara and Takahashi [9] verified that Lifshitz's theorem is valid for uniformly Lipschitzian semigroup in Hilbert spaces.

Mizoguchi and Takahashi [14] introduced the notion of a submean on an appropriate space and, using a submean, they proved a fixed point theorem for uniformly Lipschitzian semigroup in a Hilbert space. Recently, Tan and $\mathrm{Xu}$ [21] generalized the result of Mizoguchi and Takahashi [14] to a Banach space setting and, also, proved a new fixed point theorem for uniformly $k$-Lipschitzian semigroup in a uniformly convex Banach space.

Now, we consider the following class of mappings, which we call generalized Lipschitzian mapping whose $n$th iterate $T^{n}$ satisfies the following condition:

$$
\begin{aligned}
\left\|T^{n} x-T^{n} y\right\| \leq & a_{n}\|x-y\|+b_{n}\left(\left\|x-T^{n} x\right\|+\left\|y-T^{n} y\right\|\right) \\
& +c_{n}\left(\left\|x-T^{n} y\right\|+\left\|y-T^{n} x\right\|\right)
\end{aligned}
$$

for each $x, y \in K$ and $n \geq 1$, where $a_{n}, b_{n}, c_{n}$ are the nonnegative constants such that there exists an integer $n_{0}$ such that $b_{n}+c_{n}<1$ for all $n \geq n_{0}$. 
This class of generalized Lipschitzian mappings are more general than nonexpansive, asymptotically nonexpansive, Lipschitzian, and uniformly $k$-Lipschitzian mappings and it can be seen by taking $b_{n}=c_{n}=0$.

In this paper, we prove some fixed point theorems for generalized Lipschitzian semigroups in $p$-uniformly convex Banach spaces and in uniformly convex Banach spaces. Next, we give its corollaries in a Hilbert space, in $L^{p}$ spaces, in Hardy space $H^{p}$, and in Sobolev spaces $H^{k, p}$, for $1<p<\infty$ and $k \geq 0$. Our results improve and extend results from $[9,14,21,22]$.

2. Preliminaries. Let $p>1$ and denote by $\lambda$ the number in $[0,1]$ and by $w_{p}(\lambda)$ the function $\lambda \cdot(1-\lambda)^{p}+\lambda^{p} \cdot(1-\lambda)$. The functional $\|\cdot\|^{p}$ is said to be uniformly convex (cf. Zalinescu [24]) on the Banach space $E$ if there exists a positive constant $c_{p}$ such that, for all $\lambda \in[0,1]$ and $x, y \in E$, the following inequality holds:

$$
\|\lambda x+(1-\lambda) y\|^{p} \leq \lambda\|x\|^{p}+(1-\lambda)\|y\|^{p}-w_{p}(\lambda) \cdot c_{p} \cdot\|x-y\|^{p} .
$$

$\mathrm{Xu}$ [23] proved that the functional $\|\cdot\|^{p}$ is uniformly convex on the whole Banach space $E$ if and only if $E$ is $p$-uniformly convex, i.e., there exists a constant $c_{p}>0$ such that the modulus of convexity (see [7]) $\delta_{E}(\epsilon) \geq c_{p} \cdot \epsilon^{p}$ all $0 \leq \epsilon \leq 2$.

Let $G$ be a semitopological semigroup, i.e., a semigroup with a Hausdorff topology such that, for each $a \in G$, the mapping $t \rightarrow a \cdot t$ and $t \rightarrow t \cdot a$ from $G$ onto itself are continuous. A semitopological semigroup $G$ is left reversible if any two closed right ideals of $G$ have nonempty intersection. In this case, $(G, \preceq)$ is a directed system when the binary relation " $\preceq$ " on $G$ is defined by $a \preceq b$ if and only if $\{a\} \cup \overline{a G} \supseteq$ $\{b\} \cup \overline{b G}$, where $\bar{D}$ is the closure of set $D$. Examples of left reversible semigroups include commutative and all left amenable semigroups.

Let $m(G)$ be the Banach space of bounded real valued functions on $G$ with the supremum norm. Suppose $X$ is a subspace of $m(G)$ containing constants. Following Mizoguchi and Takahashi [14], we say that a real valued function $\mu$ on $X$ is a submean on $X$ if the following conditions are satisfied:

(i) $\mu(f+g) \leq \mu(f)+\mu(g)$ for all $f, g \in X$;

(ii) $\mu(\alpha f)=\alpha \mu(f)$ for all $f \in X$ and $\alpha \geq 0$;

(iii) if $f, g \in X$ with $f \leq g$, then $\mu(f) \leq \mu(g)$; and

(iv) $\mu(c)=c$ for every constant $c$.

If $\mu$ is a submean on $X$ and $f \in X$, then we denote by either $\mu(f)$ or $\mu_{t}(f(t))$, according to time and circumstances, the value of $\mu$ at $f$. For $a \in G$ and $f \in m(G)$, we define $\left(l_{a} f\right)(t)=f(a t)$ and $\left(r_{a} f\right)(t)=f(t a)$ for all $t \in G$. Let $X$ be a subspace of $m(G)$ containing constants which is $l_{G}$-invariant, i.e., $l_{a}(X) \subseteq X$ for all $a \in G$. Then a submean $\mu$ on $x$ is said to be left invariant if $\mu(f)=\mu\left(l_{a} f\right)$ for every $a \in G$ and $f \in X$. A right invariant submean is defined similarly. A submean is called invariant if it is left and right invariant. Let $K$ be a closed convex subset of a Banach space $E$. Then a collection $\mathscr{S}=\left\{T_{s}: s \in G\right\}$ of mappings of $K$ into itself is said to be a generalized Lipschitzian semigroup on $K$ if the following conditions are satisfied:

(i) $T_{s t} x=T_{s} T_{t} x$ for all $s, t \in G$ and $x \in K$;

(ii) for each $x \in K$, the mapping $t \rightarrow T_{t} x$ from $G$ into $K$ is continuous; and 
(iii) for each $s \in G$

$$
\left\|T_{s} x-T_{s} y\right\| \leq a_{s}\|x-y\|+b_{s}\left(\left\|x-T_{s} x\right\|+\left\|y-T_{s} y\right\|\right)+c_{s}\left(\left\|x-T_{s} y\right\|+\left\|y-T_{s} x\right\|\right),
$$

for $x, y \in K$, where $a_{s}, b_{s}, c_{s}>0$ such that there exists a $t_{1} \in G$ such that $b_{s}+c_{s}<1$ for all $s \geq t_{1}$.

The following lemma is needed to prove the main result:

LEMMA 1 [22, Lem. 2.1]. Let $E$ be a p-uniformly convex Banach space, $K$ a nonempty closed convex subset of $E$, and $\left\{x_{t}: t \in G\right\}$ a bounded family of elements of $E$. Also, suppose that for every $x$ in $K$, the function $f$ on $G$, defined by

$$
f(t)=\left\|x_{t}-x\right\|^{p}, \quad t \in G,
$$

belongs to X. Set

$$
r(x)=\mu_{t}\left\|x_{t}-x\right\|^{p}, \quad x \in K
$$

and

$$
r=\inf \{r(x): x \in K\}
$$

Then there exists a unique point $z$ in $K$ such that

$$
r+c_{p}\|z-x\|^{p} \leq r(x)
$$

for all $x$ in $K$, where $c_{p}$ is the constant appearing in (3).

3. Main results. Now, we prove the first result of this paper.

THEOREM 1. Let $K$ be a nonempty closed convex subset of a p-uniformly convex Banach space $E, X$ an $l_{G}$-invariant subspace of $m(G)$ containing constants which has left invariant submean $\mu$, and $\mathscr{Y}=\left\{T_{s}: s \in G\right\}$ a generalized Lipschitzian semigroup on $K$. Suppose that there exists an $x_{0}$ in $K$ such that $\left\{T_{s} x_{0}: x \in G\right\}$ is bounded and that, for every $u, v \in K$, the function $f$ on $G$ defined by

$$
f(t)=\left\|T_{t} u-v\right\|^{p}, \quad t \in G,
$$

and the function $g$ on $G$ defined by

$$
g(t)=2^{p-1}\left(\alpha_{t}^{p}+\beta_{t}^{p}\right), \quad t \in G
$$

belong to $X$. Then, if $2^{p-1}\left\{\mu_{t}\left(\alpha_{t}^{p}+\beta_{t}^{p}\right)\right\}<1+c_{p}$, where $\alpha_{t}=\left(a_{t}+b_{t}+c_{t}\right) /\left(1-b_{t}-\right.$ $\left.c_{t}\right), \beta_{t}=\left(2 b_{t}+2 c_{t}\right) /\left(1-b_{t}-c_{t}\right)$, and $c_{p}$ is the constant appearing in (3), there exists $a$ $z \in K$ such that $T_{s} z=z$ for all $s \in G$. 
Proof. Since $\left\{T_{s} x_{0}: s \in G\right\}$ is bounded, it follows that $\left\{T_{s} x: s \in G\right\}$ is bounded for every $x \in K$. By Lemma 1 , we inductively construct a sequence $\left\{x_{n}\right\}_{n=1}^{\infty}$ in $K$ in the following manner:

$$
\mu_{t}\left\|T_{t} x_{n-1}-x_{n}\right\|^{p}=\min _{y \in K} \mu_{t}\left\|T_{t} x_{n-1}-y\right\|^{p}
$$

for $n=1,2, \ldots$. It follows from Lemma 1 that

$$
c_{p}\left\|x_{n}-y\right\|^{p} \leq \mu_{t}\left\|T_{t} x_{n-1}-y\right\|^{p}-\mu_{t}\left\|T_{t} x_{n-1}-x_{n}\right\|^{p}
$$

for all $y \in K$ and $n \geq 1$. Since $T$ is generalized Lipschitzian, we get, after a simple calculation,

$$
\left\|T_{s} x-T_{s} y\right\| \leq \alpha_{s}\|x-y\|+\beta_{s}\left\|y-T_{s} y\right\|
$$

for each $x, y \in K$ and $s \in G$, where $\alpha_{s}=\left(a_{s}+b_{s}+c_{s}\right) /\left(1-b_{s}-c_{s}\right)$ and $\beta_{s}=\left(2 b_{s}+\right.$ $\left.2 c_{s}\right) /\left(1-b_{s}-c_{s}\right)$. By putting $y=T_{s} x_{n}$ into (12), we have

$$
\begin{aligned}
c_{p}\left\|x_{n}-T_{s} x_{n}\right\|^{p} & \leq \mu_{t}\left\|T_{t} x_{n-1}-T_{s} x_{n}\right\|^{p}-\mu_{t}\left\|T_{t} x_{n-1}-x_{n}\right\|^{p} \\
& =\mu_{t}\left\|T_{s t} x_{n-1}-T_{s} x_{n}\right\|^{p}-\mu_{t}\left\|T_{t} x_{n-1}-x_{n}\right\|^{p} \\
& =\mu_{t}\left\|T_{s} T_{t} x_{n-1}-T_{s} x_{n}\right\|^{p}-\mu_{t}\left\|T_{t} x_{n-1}-x_{n}\right\|^{p} \\
& \leq \mu_{t}\left[\alpha_{s}\left\|T_{t} x_{n-1}-x_{n}\right\|+\beta_{s}\left\|x_{n}-T_{s} x_{n}\right\|\right]^{p}-\mu_{t}\left\|T_{t} x_{n-1}-x_{n}\right\|^{p}
\end{aligned}
$$

or

$$
\left(c_{p}-2^{p-1} \beta_{s}^{p}\right)\left\|x_{n}-T_{s} x_{n}\right\|^{p} \leq\left(2^{p-1} \alpha_{s}^{p}-1\right) \cdot \mu_{t}\left\|T_{t} x_{n-1}-x_{n}\right\|^{p} .
$$

Therefore, we have

$$
\mu_{s}\left\|x_{n}-T_{s} x_{n}\right\|^{p} \leq A \cdot \mu_{t}\left\|T_{t} x_{n-1}-x_{n}\right\|^{p},
$$

where $A=\left(2^{p-1} \alpha_{s}^{p}-1\right) /\left(c_{p}-2^{p-1} \beta_{s}^{p}\right)<1$ by the assumption of the theorem. Since

$$
\mu_{t}\left\|T_{t} x_{n-1}-x_{n}\right\|^{p} \leq \mu_{t}\left\|T_{t} x_{n-1}-x_{n-1}\right\|^{p}
$$

by (11), it follows from (13) that

$$
\begin{aligned}
\mu_{t}\left\|T_{t} x_{n-1}-x_{n}\right\|^{p} & \leq A \cdot \mu_{t}\left\|T_{t} x_{n-1}-x_{n-1}\right\|^{p} \\
& \leq A^{n} \mu_{t}\left\|T_{t} x_{0}-x_{0}\right\|^{p} .
\end{aligned}
$$

Noticing that

$$
\left\|x_{n}-x_{n-1}\right\|^{p} \leq 2^{p-1}\left(\left\|x_{n}-T_{t} x_{n-1}\right\|^{p}+\left\|T_{t} x_{n-1}-x_{n-1}\right\|^{p}\right),
$$

we get

$$
\begin{aligned}
\left\|x_{n}-x_{n-1}\right\|^{p} & \leq 2^{p-1}\left(\mu_{t}\left\|x_{n}-T_{t} x_{n-1}\right\|^{p}+\mu_{t}\left\|T_{t} x_{n-1}-x_{n-1}\right\|^{p}\right) \\
& \leq 2^{p} \mu_{t}\left\|T_{t} x_{n-1}-x_{n-1}\right\|^{p} \\
& \leq 2^{p} A^{n-1} \mu_{t}\left\|T_{t} x_{0}-x_{0}\right\|^{p},
\end{aligned}
$$


which shows that $\left\{x_{n}\right\}$ is a Cauchy sequence and, hence, convergent. Let $z=\lim _{n \rightarrow \infty} x_{n}$. Then, for each $s \in G$, we have

$$
\begin{aligned}
\left\|z-T_{s} z\right\|^{p} & \leq\left(\left\|z-x_{n}\right\|+\left\|x_{n}-T_{s} x_{n}\right\|+\left\|T_{s} x_{n}-T_{s} z\right\|\right)^{p} \\
& \leq\left[\left(1+\alpha_{s}\right)\left\|z-x_{n}\right\|+\left(1+\beta_{s}\right)\left\|x_{n}-T_{s} x_{n}\right\|\right]^{p} \\
& \leq 2^{p-1}\left[\left(1+\alpha_{s}\right)^{p}\left\|z-x_{n}\right\|+\left(1+\beta_{s}\right)^{p} \cdot A \cdot \mu_{t}\left\|x_{n}-T_{s} x_{n}\right\|^{p}\right] \\
& \longrightarrow 0 \text { as } n \longrightarrow \infty .
\end{aligned}
$$

Therefore, $T_{s} z=z$ for all $s \in G$ and the proof is complete.

Let $E$ be a Banach space, $K$ a nonempty closed convex subset of $E$, and $G$ an unbounded subset of $[0, \infty)$ such that

$$
t+h \in G \text { for all } t, h \in G
$$

and

$$
t-h \in G \text { for all } t, h \in G \text { with } t>h
$$

(e.g., $G=[0, \infty)$ or $G=N$, the set of nonnegative integers). Suppose $\mathscr{S}=\left\{T_{s}: s \in G\right\}$ is a generalized uniformly Lipschitzian semigroup on $K$, i.e., a family of self-mappings of $K$ satisfying the conditions:

(i) $T_{s+h} x=T_{s} T_{h} x$ for all $s, h \in G$ and $x \in K$;

(ii) for each $x \in K$, the mappings $s \rightarrow T_{s} x$ from $G$ onto $K$ is continuous when $G$ has the relative topology of $[0, \infty)$; and (iii)

$$
\left\|T_{s} x-T_{s} y\right\| \leq a\|x-y\|+b\left(\left\|x-T_{s} x\right\|+\left\|y-T_{s} y\right\|\right)+c\left(\left\|x-T_{s} y\right\|+\left\|y-T_{s} x\right\|\right)
$$

for all $x, y$ in $K$ and $s$ in $G$, where $a, b, c$ are nonnegative constants such that $b+c<1$.

For the rest of this paper, $\lim _{t}$ and $\overline{\lim }_{t}$ always stand for $\lim _{t \rightarrow \infty, t \in G}, \overline{\lim }_{t \rightarrow \infty, t \in G}$ respectively.

The normal structure coefficient $N(E)$ of $E$ is defined (cf. [2]) by

$$
\begin{array}{r}
N(E)=\inf \left\{\frac{\operatorname{diam} K}{r_{K}(K)}: K \text { is a bounded convex subset of } E\right. \\
\text { consisting of more than one point }\},
\end{array}
$$

where $\operatorname{diam} K=\sup \{\|x-y\|: x, y \in K\}$ is the diameter of $K$ and $r_{K}(K)=\inf _{x \in K}$ $\left\{\sup _{y \in K}\|x-y\|\right\}$ is the Chebyshev radius of $K$ relative to itself. $E$ is said to have uniformly normal structure if $N(E)>1$. It is known that a uniformly convex Banach space has the uniformly normal structure and for a Hilbert space $H, N(H)=\sqrt{2}$. Recently, Pichugov [15] (cf. Prus [17]) showed that

$$
N\left(L^{p}\right)=\min \left\{2^{1 / p}, 2^{(p-1) / p}\right\}, \quad 1<p<\infty .
$$

Some estimate for normal structure coefficient in other Banach spaces may be found in [18]. 
Suppose $E$ is a uniformly convex Banach space. Then it is easily seen that the equation

$$
\xi^{2} \delta_{E}^{-1}\left(1-\frac{1}{\xi}\right) \tilde{N}(E)=1
$$

has a unique solution $\xi>1$, where $\tilde{N}(E)=N(E)^{-1}$.

Now, recall the definition of an asymptotic center. Let $K$ be a nonempty closed convex subset of a Banach space $E$ and $\left\{x_{t}: t \in G\right\}$ be a bounded family of elements of $E$. Then the asymptotic radius and asymptotic center of $\left\{x_{t}\right\}_{t \in G}$ with respect to $K$ are the number

$$
r_{K}\left(\left\{x_{t}\right\}\right)=\inf _{y \in K} \varlimsup_{t}\left\|x_{t}-y\right\|
$$

and the (possibly empty) set

$$
A_{K}\left(\left\{x_{t}\right\}\right)=\left\{y \in K: \varlimsup_{t}\left\|x_{t}-y\right\|=r_{K}\left(\left\{x_{t}\right\}\right)\right\},
$$

respectively. It is easy to see that if $E$ is reflexive, then $A_{K}\left(\left\{x_{t}\right\}\right)$ is nonempty bounded closed and convex and if $E$ is uniformly convex, then $A_{K}\left(\left\{x_{t}\right\}\right)$ consists of a single point.

We need the following lemma to prove our next theorem.

LEMMA 2 [22, Lem. 3.4]. Let $E$ be a Banach space with uniformly normal structure. Then for every bounded family $\left\{x_{t}\right\}_{t \in G}$ of elements of $E$, there exists $y$ in $\overline{\mathrm{co}}\left(\left\{x_{t}: t \in\right.\right.$ G\}) such that

$$
\varlimsup_{t}\left\|x_{t}-y\right\| \leq \tilde{N}(E) A\left(\left\{x_{t}\right\}\right),
$$

where $\overline{\mathrm{co}}(D)$ is the closure of the convex hull of $D \subseteq E$ and

$$
A\left(\left\{x_{t}\right\}\right)=\lim _{t}\left(\sup \left\{\left\|x_{i}-x_{j}\right\|: t \leq i, j \in G\right\}\right)
$$

is the asymptotic diameter of $\left\{x_{t}\right\}$.

Now, we are in position to prove our next theorem.

THEOREM 2. Let E be a uniformly convex Banach space, $K$ a nonempty closed convex subset of $E$, and $\mathscr{Y}=\left\{T_{s}: s \in G\right\}$ a generalized uniformly Lipschitzian semigroup on $K$ with $(\alpha+\beta)<\xi$, where $\xi>1$ is the unique solution of $(27), \alpha=(a+b+c) /(1-b-c)$ and $\beta=(2 b+2 c) /(1-b-c)$. Suppose there is an $x_{0}$ in $K$ such that $\left\{T_{s} x_{0}: s \in G\right\}$ is bounded. Then there exists $z$ in $K$ such that $T_{s} z=z$ for all $s$ in $G$.

Proof. By induction, we define a sequence $\left\{x_{n}\right\}_{0}^{\infty}$ in $K$ in the following manner:

$$
x_{n+1}=A_{K}\left(\left\{T_{t} x_{n}\right\}_{t \in G}\right)
$$

for $n=0,1, \ldots$, i.e., $x_{n+1}$ is the unique point in $K$ such that

$$
\varlimsup_{t}\left\|T_{t} x_{n}-x_{n+1}\right\|=\inf _{y \in K} \varlimsup_{t}\left\|T_{t} x_{n}-y\right\| .
$$


Write $r_{n}=r_{K}\left(\left\{T_{t} x_{n}\right\}_{t \in G}\right)$. Then by Lemma 2, we have

$$
\begin{aligned}
r_{n} & =\varlimsup_{t}\left\|T_{t} x_{n}-x_{n-1}\right\| \\
& \leq \tilde{N}(E) \cdot A\left(\left\{T_{t} x_{n}\right\}_{t \in G}\right) \\
& =\tilde{N}(E) \lim _{t}\left(\sup \left\{\left\|T_{i} x_{n}-T_{j} x_{n}\right\|: t \leq i, j \in G\right\}\right) \\
& \leq \tilde{N}(E)(\alpha+\beta) \cdot d\left(x_{n}\right),
\end{aligned}
$$

that is,

$$
r_{n} \leq(\alpha+\beta) \cdot \tilde{N}(E) d\left(x_{n}\right)
$$

where $d\left(x_{n}\right)=\sup \left\{\left\|T_{t} x_{n}-x_{n}\right\|: t \in G\right\}$. We may assume that $d\left(x_{n}\right)>0$ for all $n \geq 0$. Let $n \geq 0$ be fixed and let $\epsilon>0$ be small enough. First, choose $j \in G$ such that

$$
\left\|T_{j} x_{n+1}-x_{n+1}\right\|>d\left(x_{n+1}\right)-\epsilon
$$

and then choose $s_{0}$ in $G$ so large that

$$
\left\|T_{s} x_{n}-x_{n+1}\right\|<r_{n}+\epsilon
$$

and

$$
\left\|T_{s} x_{n}-T_{j} x_{n+1}\right\| \leq \alpha\left\|T_{s-j} x_{n}-x_{n+1}\right\|+\beta\left\|T_{j} x_{n}-x_{n}\right\| \leq(\alpha+\beta)\left(r_{n}+\epsilon\right)
$$

for all $s \geq s_{0}$. It, then, follows that

$$
\left\|T_{s} x_{n}-\frac{1}{2}\left(x_{n+1}+T_{j} x_{n+1}\right)\right\| \leq(\alpha+\beta)\left(r_{n}+\epsilon\right)\left(1-\delta_{E}\left(\frac{d\left(x_{n+1}\right)-\epsilon}{(\alpha+\beta)\left(r_{n}+\epsilon\right)}\right)\right)
$$

for $s \geq s_{0}$ and, hence,

$$
\begin{aligned}
r_{n} & \leq \varlimsup_{s}\left\|T_{s} x_{n}-\frac{1}{2}\left(x_{n+1}+T_{j} x_{n+1}\right)\right\| \\
& \leq(\alpha+\beta)\left(r_{n}+\epsilon\right)\left(1-\delta_{E}\left(\frac{d\left(x_{n+1}\right)-\epsilon}{(\alpha+\beta)\left(r_{n}+\epsilon\right)}\right)\right) .
\end{aligned}
$$

Taking the limit as $\epsilon \longrightarrow 0$, we get

$$
r_{n} \leq(\alpha+\beta) \cdot r_{n}\left(1-\delta_{E}\left(\frac{d\left(x_{n+1}\right)}{(\alpha+\beta) r_{n}}\right)\right)
$$

which together with (35) leads to the conclusion

$$
d\left(x_{n+1}\right) \leq(\alpha+\beta)^{2} \tilde{N}(E) \delta_{E}^{-1}\left(1-\frac{1}{(\alpha+\beta)}\right) d\left(x_{n}\right) .
$$

Hence,

$$
d\left(x_{n}\right) \leq A d\left(x_{n-1}\right) \leq A^{n} d\left(x_{0}\right),
$$


where $A=(\alpha+\beta)^{2} \tilde{N}(E) \delta_{E}^{-1}(1-(1 /(\alpha+\beta)))<1$ by assumption. Noticing that

$$
\begin{aligned}
\left\|x_{n+1}-x_{n}\right\| & \leq \varlimsup_{t}\left\|T_{t} x_{n}-x_{n+1}\right\|+\varlimsup_{t}\left\|T_{t} x_{n}-x_{n}\right\| \\
& \leq r_{n}+d\left(x_{n}\right) \leq 2 d\left(x_{n}\right),
\end{aligned}
$$

we see from (43) that $\left\{x_{n}\right\}$ is a Cauchy sequence and, hence, strongly convergent. Let $z=\lim _{n} x_{n}$. Then we have, for each $s \in G$,

$$
\begin{aligned}
\left\|z-T_{s} z\right\| & \leq\left\|z-x_{n}\right\|+\left\|T_{s} x_{n}-x_{n}\right\|+\left\|T_{s} x_{n}-T_{s} z\right\| \\
& \leq(1+\alpha)\left\|z-x_{n}\right\|+(1+\beta) d\left(x_{n}\right) \\
& \longrightarrow 0 \text { as } n \rightarrow \infty .
\end{aligned}
$$

This completes the proof.

As a consequence of Theorem 2, we have the following result.

COROLLARY 1. Let $K$ be a nonempty bounded closed convex subset of a uniformly convex Banach space $E$ and let $T: K \longrightarrow K$ be a generalized uniformly Lipschitzian mapping with $(\alpha+\beta)<\xi$ ( $\xi$ is as in Theorem 2). Then $T$ has a fixed point.

If we take $b=c=0$ in Theorem 2, then we have the following result from Theorem 2:

COROllary 2 [22, Thm. 3.5]. Let $E$ be a uniformly convex Banach space, $K$ a nonempty closed convex subset of $E$, and $\mathscr{S}=\left\{T_{s}: s \in G\right\}$ a uniformly $k$-Lipschitzian semigroup on $K$ with $k<\xi$, where $\xi>1$ is the unique solution of (27). Suppose there is an $x_{0}$ in $K$ such that $\left\{T_{s} x_{0}: s \in G\right\}$ is bounded. Then there exists $z$ in $K$ such that $T_{s} z=z$ for all $s$ in $G$.

4. Some applications. Since a Hilbert space $H$ is 2-uniformly convex and the following equality holds:

$$
\|\lambda x+(1-\lambda) y\|^{2}=\lambda\|x\|^{2}+(1-\lambda)\|y\|^{2}-\lambda(1-\lambda)\|x-y\|^{2}
$$

for all $x, y$ in $H$ and $\lambda \in[0,1]$.

By Theorem 1 and (46), we immediately obtain the following:

COROLLARY 3. Let E be a nonempty closed convex subset of a Hilbert space $H, X$ be an $l_{G}$-invariant subspace of $m(G)$ containing constants which has left invariant submean $\mu$, and $\mathscr{S}=\left\{T_{s}: s \in G\right\}$ be a generalized Lipschitzian semigroup on K. Suppose that there exists an $x_{0}$ in $K$ such that $\left\{T_{s} x_{0}: s \in G\right\}$ is a generalized Lipschitzian semigroup on $K$. Suppose that there exists an $x_{0}$ in $K$ such that $\left\{T_{s} x_{0}: s \in G\right\}$ is bounded and that for every $u, v$ in $K$, then the function $f$ on $G$ defined by

$$
f(t)=\left\|T_{t} u-v\right\|^{2}, \quad t \in G
$$

and the function $g$ on $G$ defined by

$$
g(t)=2\left(\alpha_{t}^{2}+\beta_{t}^{2}\right), \quad t \in G
$$

belong to $X$. Then, if $\left\{\mu_{t}\left(\alpha_{t}^{2}+\beta_{t}^{2}\right)\right\}<1$, where $\alpha_{t}=\left(a_{t}+b_{t}+c_{t}\right) /\left(1-b_{t}-c_{t}\right)$ and $\beta_{t}=\left(2 b_{t}+2 c_{t}\right) /\left(1-b_{t}-c_{t}\right)$, there exists $z$ in $K$ such that $T_{s} z=z$ for all $s$ in $G$. 
If $1<p \leq 2$, then we have for all $x, y$ in $L^{p}$ and $\lambda \in[0,1]$

$$
\|\lambda x+(1-\lambda) y\|^{2} \leq \lambda\|x\|^{2}+(1-\lambda)\|y\|^{2}-\lambda(1-\lambda)(p-1)\|x-y\|^{2}
$$

(the inequality (49) is contained in [12, 20]).

Assume that $2<p<\infty$ and $t_{p}$ is the unique zero of the function $g(x)=-x^{p-1}+$ $(p-1) x+p-2$ in the interval $(1, \infty)$. Let

$$
c_{p}=(p-1)\left(1+t_{p}\right)^{2-p}=\frac{1+t_{p}^{p-1}}{\left(1+t_{p}\right)^{p-1}} .
$$

Then we have the following inequality

$$
\|\lambda x+(1-\lambda) y\|^{p} \leq \lambda\|x\|^{p}+(1-\lambda)\|y\|^{p}-w_{p}(\lambda) \cdot c_{p} \cdot\|x-y\|^{p}
$$

for all $x, y$ in $L^{p}$ and $\lambda \in[0,1]$. (The inequality (51) is essentially due to Lim [11].)

By Theorem 1 and inequality (49) and (51), we immediately obtain the following result.

COROLlary 4. Let $K$ be a closed convex subset of an $L^{p}$ space, $1<p<\infty, X$ be an $l_{G^{-}}$ invariant subspace of $m(G)$ containing constants which has a left invariant submean $\mu$, and $\mathscr{Y}=\left\{T_{s}: s \in G\right\}$ be a generalized Lipschitzian semigroup on $K$. Suppose that $\left\{T_{s} x_{0}: s \in G\right\}$ is bounded for some $x_{0} \in K$ and that for every $u, v$ in $K$, the functions $f$ and $g$ on $G$ defined as in Theorem 1 belong to $X$. If $2 \mu_{s}\left(\alpha_{s}^{2}+\beta_{s}^{2}\right)<p$ when $1<p \leq 2$ and $2^{p-1} \mu_{s}\left(\alpha_{s}^{p-1}+\beta_{s}^{p-1}\right)<1+c_{p}$ when $p>2$, where $\alpha_{s}=\left(a_{s}+b_{s}+c_{s}\right) /\left(1-b_{s}-c_{s}\right)$ and $\beta_{s}=\left(2 b_{s}+2 c_{s}\right) /\left(1-b_{s}-c_{s}\right)$, then there exists $z \in K$ such that $T_{s} z=z$ for all $s \in G$.

Let $H^{p}, 1<p<\infty$, denote the Hardy space [5] of all functions $x$ analytic in the unit disk $|z|<1$ of the complex plane and such that

$$
\|x\|=\lim _{r \rightarrow 1^{-}}\left(\frac{1}{2 \pi} \int_{0}^{2 \pi}\left|x\left(r e^{i \theta}\right)\right|^{p} d \theta\right)^{1 / p}<\infty .
$$

Now, let $\Omega$ be an open subset of $\mathbb{R}^{n}$. Denote by $H^{k, p}(\Omega), k \geq 0,1<p<\infty$, the Sobolev space [1, p. 149] of distribution $x$ such that $D^{\alpha} x \in L^{p}(\Omega)$ for all $|\alpha|=a_{1}+\cdots+\alpha_{n} \leq k$ equipped with the norm

$$
\|x\|=\left(\sum_{|\alpha| \leq k} \int_{\Omega}\left|D^{\alpha} x(\omega)\right|^{p} d \omega\right)^{1 / p} .
$$

Let $\left(\Omega_{\alpha}, \Sigma_{\alpha}, \mu_{\alpha}\right), \alpha \in \wedge$, be a sequence of positive measure spaces, where index set $\wedge$ is finite or countable. Given a sequence of linear subspaces $X_{\alpha}$ in $L^{p}\left(\Omega_{\alpha}, \Sigma_{\alpha}, \mu_{\alpha}\right)$, we denote by $L_{q, p}, 1<p<\infty$ and $q=\max \{2, p\}$ [13], the linear space of all sequences $x=\left\{x_{\alpha} \in X_{\alpha}: \alpha \in \wedge\right\}$ equipped with the norm

$$
\|x\|=\left(\sum_{\alpha \in \wedge}\left(\left\|x_{\alpha}\right\|_{p, \alpha}\right)^{q}\right)^{1 / q}
$$


where $\|\cdot\|_{p, \alpha}$ denotes the norm in $L^{p}\left(\Omega_{\alpha}, \Sigma_{\alpha}, \mu_{\alpha}\right)$.

Finally, let $L_{p}=\left(S_{1}, \Sigma_{1}, \mu_{1}\right)$ and $L_{q}=\left(S_{2}, \Sigma_{2}, \mu_{2}\right)$, where $1<p<\infty, q=\max \{2, p\}$ and $\left(S_{i}, \Sigma_{i}, \mu_{i}\right)$ are positive measure spaces. Denote by $L_{q}\left(L_{p}\right)$ the Banach spaces [4, III.2.10] of all measurable $L_{p}$-value function $x$ on $S_{2}$ such that

$$
\|x\|=\left(\int_{S_{2}}\left(\|x(S)\|_{p}\right)^{q} \mu_{2}(d s)\right)^{1 / q} .
$$

These spaces are $q$-uniformly convex with $q=\max \{2, p\}[16,19]$ and the norm in these spaces satisfies

$$
\|\lambda x+(1-\lambda) y\|^{q} \leq \lambda\|x\|^{q}+(1-\lambda)\|y\|^{q}-d \cdot w_{q}(\lambda) \cdot\|x-y\|^{q}
$$

with a constant

$$
d=d_{p}= \begin{cases}\frac{p-1}{8} & \text { for } 1<p \leq 2 \\ \frac{1}{p \cdot 2^{p}} & \text { for } 2<p<\infty .\end{cases}
$$

Now, from Theorem 1 , we have the following result.

COROLlary 5. Let $K$ be a closed convex subset of the space $E$, where $E=H^{p}$, or $E=H^{k, p}(\Omega)$, or $E=L_{q, p}$, or $E=L_{q}\left(L_{p}\right)$, and $1<p<\infty, q=\max \{2, p\}, k \geq 0, X$ be an $l_{G}$-invariant subspace of $m(G)$ containing constants which has a left invariant submean $\mu$, and $\mathscr{S}=\left\{T_{s}: s \in G\right\}$ be a generalized Lipschitzian semigroup on $K$. Suppose that $\left\{T_{s} x_{0}: s \in G\right\}$ is bounded for some $x_{0}$ in $K$ and that for every $u, v$ in $K$, the functions $f$ and $g$ on $G$ defined as in Theorem 1 belong to $X$. If $2^{q-1} \mu_{s}\left(\alpha_{s}^{q}+\beta_{s}^{q}\right)<1+d$, where $\alpha_{s}=\left(a_{s}+b_{s}+c_{s}\right) /\left(1-b_{s}-c_{s}\right)$ and $\beta_{s}=\left(2 b_{s}+2 c_{s}\right) /\left(1-b_{s}-c_{s}\right)$, then there exists $z \in K$ such that $T_{s} z=z$ for all $s \in G$.

ACKNOWLEDgement. The second author was supported by the Basic Science Research Institute Program, Ministry of Education, Korea, 1997, Project No. BSRI-971405 .

\section{REFERENCES}

[1] J. Barros-Neto, An introduction to the theory of distributions, Pure and Applied Mathematics, vol. 14, Marcel Dekker, Inc., New York, 1973. MR 57 1113. Zbl 273.46026.

[2] W. L. Bynum, Normal structure coefficients for Banach spaces, Pacific J. Math. 86 (1980), no. 2, 427-436. MR 81m:46030. Zbl 442.46018.

[3] D. J. Downing and W. O. Ray, Uniformly Lipschitzian semigroups in Hilbert space, Canad. Math. Bull. 25 (1982), no. 2, 210-214. MR 84e:47066. Zbl 475.47045.

[4] N. Dunford and J. T. Schwartz, Linear Operators. I. General Theory, With the assistance of W. G. Bade and R. G. Bartle. Pure and Applied Mathematics, vol. 7, Interscience Publishers, Ltd., London, 1958. MR 22\#8302. Zbl 084.10402.

[5] P. L. Duren, Theory of $H^{p}$ spaces, Pure and Applied Mathematics, vol. 38, Academic Press, New York, 1970. MR 42\#3552. Zbl 215.20203.

[6] K. Goebel and W. A. Kirk, A fixed point theorem for transformations whose iterates have uniform Lipschitz constant, Studia Math. 47 (1973), 135-140. MR 491242. Zbl 265.47044.

[7] _ Topics in metric fixed point theory, Cambridge Studies in Advanced Mathematics, vol. 28, Cambridge University Press, Cambridge, 1990. MR 92c:47070. Zbl 708.47031. 
[8] K. Goebel, W. A. Kirk, and R. L. Thele, Uniformly Lipschitzian families of transformations in Banach spaces, Canad. J. Math. 26 (1974), 1245-1256. MR 5010919. Zbl 285.47039.

[9] H. Ishihara and W. Takahashi, Fixed point theorems for uniformly Lipschitzian semigroups in Hilbert spaces, J. Math. Anal. Appl. 127 (1987), no. 1, 206-210. MR 88j:47076. Zbl 637.47028.

[10] E. A. Lifshitz, A fixed point theorem for operators in strongly convex spaces, Voronež. Gos. Univ. Trudy Mat. Fak. 16 (1975), 23-28 (Russian). MR 5717401.

[11] T. C. Lim, On some $L^{p}$ inequalities in best approximation theory, J. Math. Anal. Appl. 154 (1991), no. 2, 523-528. MR 92d:41029. Zbl 744.41015.

[12] T. C. Lim, H. K. Xu, and Z. B. Xu, Some $L^{p}$ inequalities and their applications to fixed point theory and approximation theory, Progress in approximation theory (Boston), Academic Press, 1991, pp. 609-624. MR 92j:47112.

[13] J. Lindenstrauss and L. Tzafriri, Classical Banach spaces. II, Ergebnisse der Mathematik und ihrer Grenzgebiete [Results in Mathematics and Related Areas], vol. 97, Springer-Verlag, New York, 1979, Function spaces. MR 81c:46001. Zbl 403.46022.

[14] N. Mizoguchi and W. Takahashi, On the existence of fixed points and ergodic retractions for Lipschitzian semigroups in Hilbert spaces, Nonlinear Anal. 14 (1990), no. 1, 69-80. MR 91h:47071. Zbl 695.47063.

[15] S. A. Pichugov, The Jung constant of the space $L_{p}$, Mat. Zametki 43 (1988), no. 5, 604-614 (Russian), Translation:Math. Notes 43(1988),348-358. MR 90a:46037. Zbl 644.46016.

[16] B. Prus and R. Smarzewski, Strongly unique best approximations and centers in uniformly convex spaces, J. Math. Anal. Appl. 121 (1987), no. 1, 10-21. MR 88i:41052. Zbl 617.41046.

[17] S. Prus, On Bynum's fixed point theorem, Atti Sem. Mat. Fis. Univ. Modena 38 (1990), no. 2, 535-545. MR 91k:47146. Zbl 724.46020.

[18]_ Some estimates for the normal structure coefficient in Banach spaces, Rend. Circ. Mat. Palermo (2) 40 (1991), no. 1, 128-135. MR 92j:46029. Zbl 757.46029.

[19] R. Smarzewski, Strongly unique best approximation in Banach spaces. II, J. Approx. Theory 51 (1987), no. 3, 202-217. MR 88j:41064. Zbl 657.41022.

[20] _ _ On an inequality of Bynum and Drew, J. Math. Anal. Appl. 150 (1990), no. 1, 146-150. MR 91g:47046. Zbl 716.46023.

[21] K. K. Tan and H. K. Xu, Fixed point theorems for Lipschitzian semigroups in Banach spaces, Nonlinear Anal. 20 (1993), no. 4, 395-404. MR 94e:47087. Zbl 781.47044.

[22] H. K. Xu, Fixed point theorems for uniformly Lipschitzian semigroups in uniformly convex spaces, J. Math. Anal. Appl. 152 (1990), no. 2, 391-398. MR 91m:47089. Zbl 722.47050 .

[23] _ Inequalities in Banach spaces with applications, Nonlinear Anal. 16 (1991), no. 12, 1127-1138. MR 92e:47126. Zbl 757.46033.

[24] C. Zalinescu, On uniformly convex functions, J. Math. Anal. Appl. 95 (1983), no. 2, 344374. MR 85a:26018. Zbl 519.49010.

ThAKUR: GovT. B. H. S. S. GARIABAND, Dist. RAIPUR, M. P. 493889, INDIA

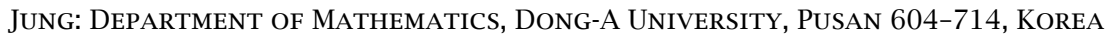

E-mail address: jungjs@seunghak.donga.ac. kr 


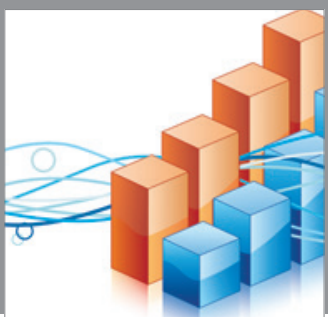

Advances in

Operations Research

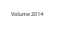

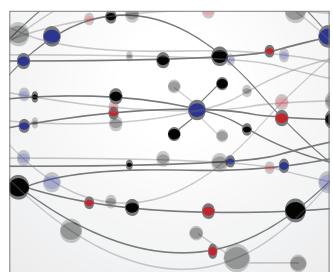

\section{The Scientific} World Journal
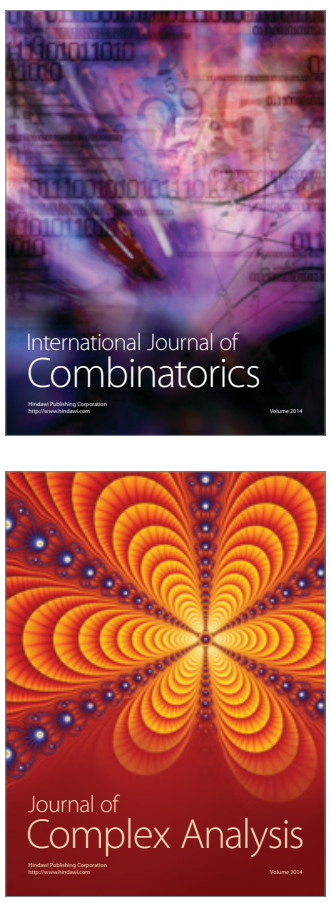

International Journal of

Mathematics and

Mathematical

Sciences
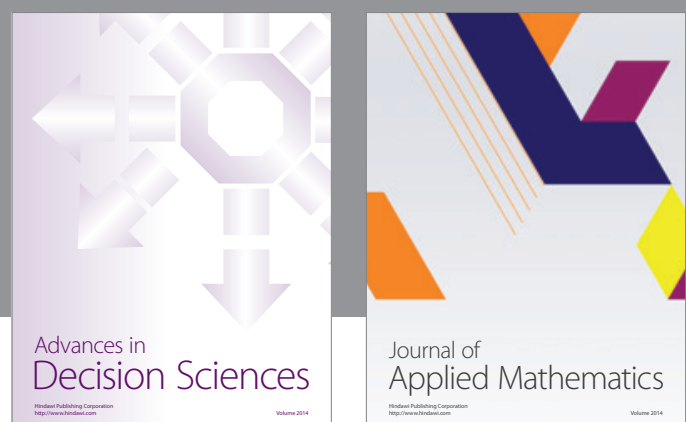

Journal of

Applied Mathematics
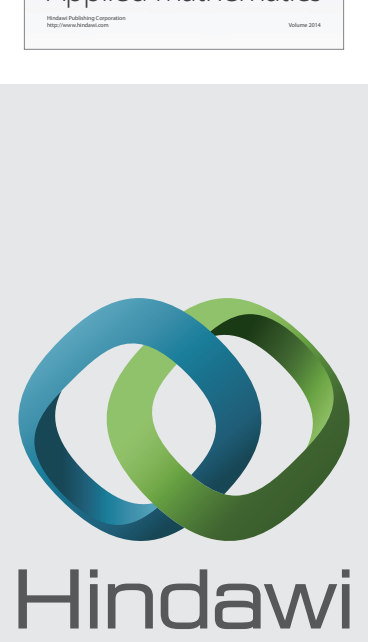

Submit your manuscripts at http://www.hindawi.com
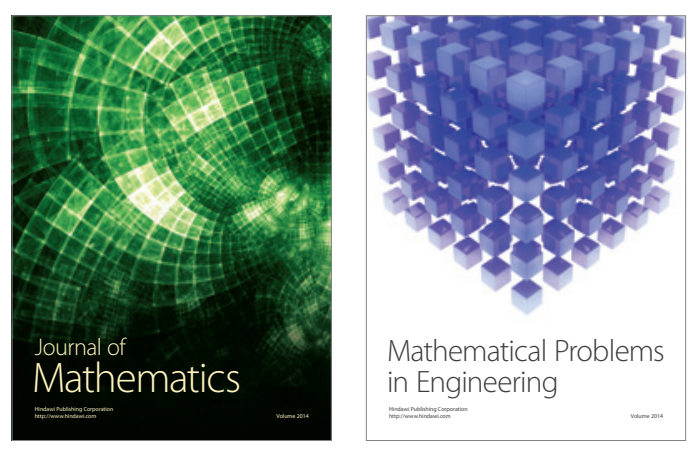

Mathematical Problems in Engineering
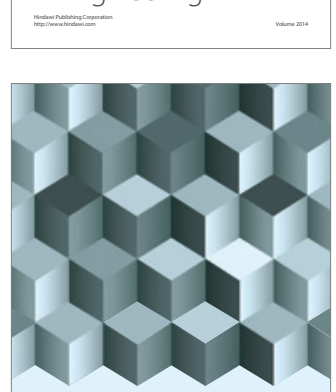

Journal of

Function Spaces
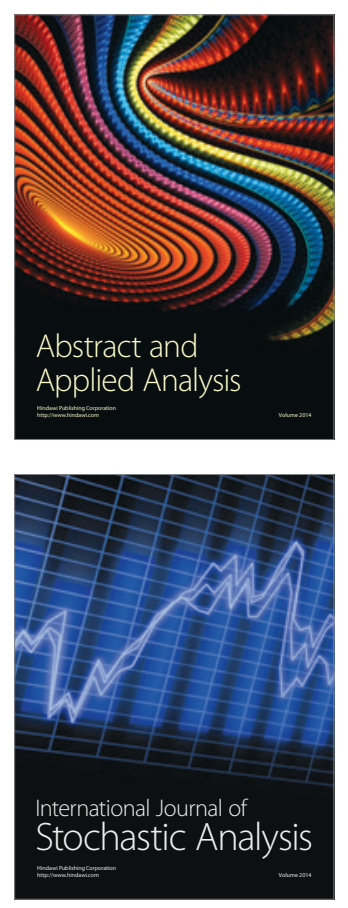

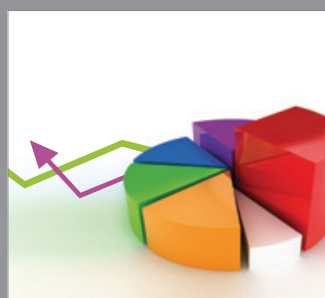

ournal of

Probability and Statistics

Promensencen
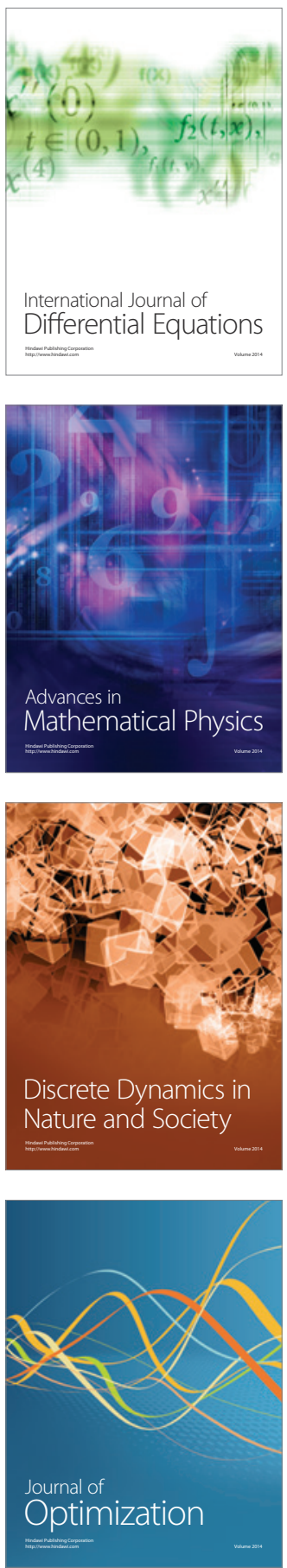